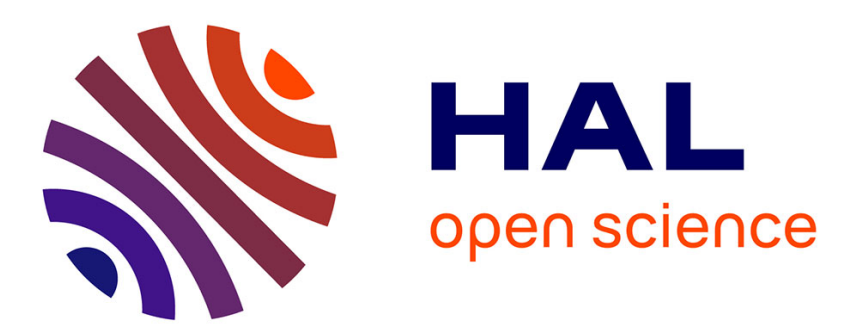

\title{
Unbounded Second-Order State-Dependent Moreau's Sweeping Processes in Hilbert Spaces
}

\author{
Samir Adly, Le Ba Khiet
}

\section{To cite this version:}

Samir Adly, Le Ba Khiet. Unbounded Second-Order State-Dependent Moreau's Sweeping Processes in Hilbert Spaces. Journal of Optimization Theory and Applications, 2016, 169, pp.407-423. 10.1007/s10957-016-0905-2 . hal-01313642

\section{HAL Id: hal-01313642 \\ https://hal.science/hal-01313642}

Submitted on 17 Feb 2017

HAL is a multi-disciplinary open access archive for the deposit and dissemination of scientific research documents, whether they are published or not. The documents may come from teaching and research institutions in France or abroad, or from public or private research centers.
L'archive ouverte pluridisciplinaire HAL, est destinée au dépôt et à la diffusion de documents scientifiques de niveau recherche, publiés ou non, émanant des établissements d'enseignement et de recherche français ou étrangers, des laboratoires publics ou privés. 


\title{
Unbounded Second-Order State-Dependent Moreau's Sweeping Processes in Hilbert Spaces
}

\author{
Samir Adly ${ }^{1} \cdot$ Ba Khiet Le ${ }^{2}$
}

\begin{abstract}
In this paper, an existence and uniqueness result of a class of second-order sweeping processes, with velocity in the moving set under perturbation in infinitedimensional Hilbert spaces, is studied by using an implicit discretization scheme. It is assumed that the moving set depends on the time, the state and is possibly unbounded. The assumptions on the Lipschitz continuity and the compactness of the moving set, and the linear growth boundedness of the perturbation force are weaker than the ones used in previous papers.
\end{abstract}

Keywords Moreau's sweeping process · Quasi-variational inequalities · Differential inclusion

\section{Introduction}

In 1971, the sweeping process was introduced and deeply studied by J. J. Moreau in a series of papers (see, e.g., [1-4]). This kind of problems plays an important role in elasto-plasticity, quasi-statics and nonsmooth dynamics with unilateral con- 
straints. Roughly speaking, a point is swept by a moving closed and convex set, which depends on time in a Hilbert space and can be formulated in the form of first-order differential inclusion involving normal cone operators. Sweeping processes represent a nice and powerful mathematical framework for many nonsmooth dynamical systems, including Lagrangian systems. There are plenty of existence and uniqueness results (see, e.g., [5-10]) for variants of first-order sweeping processes in the literature. The second-order sweeping processes have been also considered by many authors (see, e.g., [7,10-14]). In [11], Castaing studied for the first time the second-order sweeping processes, where the moving set depends on the state with convex, compact values. Let us note that the boundedness assumption on the moving set for the second-order case is essential in most previous works: see, for example, some recent papers [7,10,13,14]. In [15], Castaing et al. considered the possibly unbounded moving set satisfying the classical Lipschitz continuity assumption with respect to Hausdorff distance. However, it is difficult for unbounded set to hold this assumption since the Hausdorff distance of two unbounded sets may equal the infinity, for example, the case of rotating hyperplane. In this paper, we propose an implicit discretization scheme based on the Moreau's catching-up algorithm [3] with different techniques to analyze the second-order sweeping processes under perturbation in Hilbert spaces. The moving set depends on the time, the state and is possibly unbounded. The set is supposed to be closed, convex and to have a Lipschitz variation of intersection with some particular ball (with a Lipschitz constant depending on the radius of the ball). It is obvious that this kind of Lipschitz continuity assumption is more feasible than the classical one for the unbounded moving set. The perturbation force is supposed to be upper semicontinuous with convex and weakly compact values and only need to satisfy the weak linear growth condition (i.e., the intersection between the perturbation force and the ball with linear growth is nonempty). In addition, the compactness assumption on the moving set is weaker than the one used in previous works[7,10,13-15], since it only requires to check the Kuratowski measure of noncompactness for a fixed ball. We also consider the case when the moving set is anti-monotone (which replaces the compactness assumption) as in [14] under the current settings. Our methodology is based on convex and variational analysis $[16,17]$.

The paper is organized as follows. In Sect. 2, we recall some basic notations, definitions and useful results which are used throughout the paper. The existence and uniqueness of solutions are thoroughly analyzed in Sect. 3. Some conclusions end up the paper in Sect. 4.

\section{Notation and Preliminaries}

We begin with some notations used in the paper. Let $H$ be a real Hilbert space. Denote by $\langle\cdot, \cdot\rangle,\|\cdot\|$ the scalar product and the corresponding norm in $H$. Denote by $I$ the identity operator, by $\mathbb{B}$ the unit ball in $H$. The distance from a point $x$ to a set $K$ is denoted by $d(x, K)$. If $K$ is closed and convex, then for each $x \in H$, there exists uniquely a point $y \in K$ which is nearest to $x$ and set $y:=\operatorname{proj}(K ; x)$. The normal cone of $K$ is given by 


$$
N_{K}(x):=\{p \in H:\langle p, y-x\rangle \leq 0 \text {, for all } y \in K\} .
$$

The support function of $K$ is defined as follows

$$
\sigma(K ; z):=\sup _{x \in K}\langle x, z\rangle, z \in H .
$$

It is not difficult to see that

$$
z \in N_{K}(x) \text { if and only if } \sigma(K ; z)=\langle z, x\rangle \text { and } x \in K \text {. }
$$

The Hausdorff distance between the sets $A$ and $B$ is given by

$$
d_{H}(A ; B):=\max \{e(A, B) ; e(B, A)\}
$$

where $e(A ; B):=\sup _{x \in A} d(x, B)$ is the excess of $A$ over $B$.

A sequence $\left(x_{n}\right) \subset H$ converges weakly to $x \in H$, provided that $\left\langle x_{n}, z\right\rangle \rightarrow\langle x, z\rangle$, as $n \rightarrow+\infty$ for all $z$ in $H$. Let us recall a known property of an integral functionals with respect to the weak convergence of functions with values in $H$ (see [18, Corollary, p. 227] or [10, Lemma 3]).

Lemma 2.1 Suppose that for all $t \in[0, T]$, the set $C(t) \subset H$ is nonempty, closed, convex and satisfies

$$
d_{H}(C(t), C(s)) \leq L_{C}|t-s| \forall s, t \in[0, T]
$$

for some $L_{C}>0$. Set

$$
\Phi(v):=\int_{0}^{T} \sigma(C(s) ; v(s)) d s \text { for } v \in L^{\infty}([0, T] ; H)
$$

Then, $\Phi$ is weakly lower semicontinuous, i.e.,

$$
\Phi(v) \leq \liminf _{n \rightarrow+\infty} \Phi\left(v_{n}\right)
$$

where $v_{n} \rightarrow v$ in weak-star topology of $L^{\infty}([0, T] ; H)$.

The following lemma is a discrete version of Gronwall's inequality.

Lemma 2.2 Let $\alpha>0$ and $\left(u_{n}\right),\left(\beta_{n}\right)$ be nonnegative sequences satisfying

$$
u_{n} \leq \alpha+\sum_{k=0}^{n-1} \beta_{k} u_{k} \forall n=0,1,2, \ldots \quad\left(\text { with } \beta_{-1}:=0\right)
$$


Then, for all $n$, we have

$$
u_{n} \leq \alpha \exp \left(\sum_{k=0}^{n-1} \beta_{k}\right)
$$

Finally, we recall the Kuratowski measure of noncompactness for a bounded set $B$ in $H$, which is defined as follows

$$
\gamma(B):=\inf \left\{r>0: B=\bigcup_{i=1}^{n} B_{i} \text { for some } n \text { and } B_{i} \text { with } \operatorname{diam}\left(B_{i}\right) \leq r\right\}
$$

One has the following lemma (see, e.g., [19, Proposition 9.1]).

Lemma 2.3 Let $B_{1}$ and $B_{2}$ be bounded sets of the infinite-dimensional Hilbert space H. Then,

(i) $\gamma\left(B_{1}\right)=0 \Leftrightarrow B_{1}$ is relative compact.

(ii) If $B_{1} \subset B_{2}$, then $\gamma\left(B_{1}\right) \leq \gamma\left(B_{2}\right)$.

(iii) $\gamma\left(B_{1}+B_{2}\right) \leq \gamma\left(B_{1}\right)+\gamma\left(B_{2}\right)$.

(iv) $\gamma\left(x_{0}+r \mathbb{B}\right)=2 r$ for some $x_{0} \in H$ and $r>0$.

\section{Main Result}

In this section, the existence and uniqueness of solutions of the following second-order sweeping processes

$$
(\mathcal{S})\left\{\begin{array}{l}
\ddot{u}(t) \in-N_{C(t, u(t))}(\dot{u}(t))-F(t, u(t), \dot{u}(t)) \quad \text { a.e. } t \in[0, T], \\
u(0)=u_{0}, \dot{u}(0)=v_{0} \in C\left(0, u_{0}\right),
\end{array}\right.
$$

are analyzed thoroughly under weaker assumptions, by using an implicit discretization scheme and techniques different from previous works. The moving set $C$ is supposed to be nonempty, closed, convex and to have a Lipschitz variation of intersection with some particular ball. The perturbation force $F$ is upper semicontinuous with convex, weakly compact values and satisfies the weak linear growth condition. For details, let us make the assumptions below. Let $H$ be a real Hilbert space and let be given $u_{0} \in H, v_{0} \in C\left(0, u_{0}\right)$.

Remark 1 Let us consider the simple case when $C(\cdot)$ depends only on $t$ and is expressed in the form of unilateral inequality constraints, i.e.,

$$
C(t)=\left\{x \in \mathbb{R}^{n}: g_{1}(t, x) \leq 0, \ldots, g_{m}(t, x) \leq 0\right\},
$$

where function $g_{k}:[0, T] \times \mathbb{R}^{n} \rightarrow \mathbb{R}$ is supposed to be of class $\mathcal{C}^{1}$ for each $k=1,2, \ldots, m$. Clearly, the convexity of the functions $g_{k}$ implies the convexity of the set $C(t)$. In order to go beyond the convexity assumption of the set $C(\cdot)$, the 
class of prox-regular sets is more appropriate. However, the sublevels of prox-regular functions and levels of differentiable mappings with Lipschitz derivatives may fail to be prox-regular. We need some qualification conditions on the functions $g_{k}$ to ensure the prox-regularity of the set $C(t)$ (see [20] for more details). In the present paper, we will content ourselves with the convexity assumption.

The following assumptions will be useful.

Assumption 1 (i) For all $t \in[0, T]$ and $x \in H, C(t, x) \subset H$ is nonempty, closed, convex and there exists $L_{C}>0$ such that

$$
\Gamma(t, x, s, y) \leq L_{C}(|t-s|+\|x-y\|),
$$

for all $s, t \in[0, T]$ and $x, y \in M_{1} \mathbb{B}$ where

$$
\begin{aligned}
& \Gamma(t, x, s, y) \\
& := \begin{cases}d_{H}\left(C(t, x) \cap M_{1} \mathbb{B} ; C(s, y) \cap M_{1} \mathbb{B}\right), & \text { if } C(t, x) \cap M_{1} \mathbb{B} \neq \emptyset, C(s, y) \cap M_{1} \mathbb{B} \neq \emptyset, \\
e\left(C(t, x) \cap M_{1} \mathbb{B} ; C(s, y)\right), & \text { if } C(t, x) \cap M_{1} \mathbb{B} \neq \emptyset, C(s, y) \cap M_{1} \mathbb{B}=\emptyset, \\
0, & \text { if } C(t, x) \cap M_{1} \mathbb{B}=\emptyset, C(s, y) \cap M_{1} \mathbb{B}=\emptyset,\end{cases}
\end{aligned}
$$

and

$$
M_{1}:=1+\left\|u_{0}\right\|+\left\|v_{0}\right\|+\left(L_{C}+2 L_{F}\right) T+e^{\left(L_{C}+2 L_{F}+1\right) T} .
$$

(ii) For all $t \in[0, T], C\left(t, M_{1} \mathbb{B}\right) \cap 2 M_{1} \mathbb{B}$ is relatively compact in $H$, or equivalently

$$
\gamma\left(C\left(t, M_{1} \mathbb{B}\right) \cap 2 M_{1} \mathbb{B}\right)=0,
$$

where $\gamma$ is the Kuratowski measure of noncompactness.

Assumption 2 The set-valued mapping $F: \operatorname{gph}(C) \rightrightarrows H$ is upper semicontinuous with convex, weakly compact values in $H$ and satisfies the weak linear growth condition, i.e., there exists $L_{F}>0$ such that, for all $t \in[0, T], x \in H$ and $y \in C(t, x)$, then

$$
F(t, x, y) \cap L_{F}(1+\|x\|+\|y\|) \mathbb{B} \neq \varnothing .
$$

Here $\operatorname{gph}(C)$ denotes the graph of $C$.

Now we are ready for the main result.

Theorem 3.1 (Existence) Let $H$ be a Hilbert space and let Assumptions 1, 2 hold. Then, for given initial condition $u_{0} \in H, v_{0} \in C\left(0, u_{0}\right)$, there exists a solution $u$ in the following sense

1. $(\mathcal{S})$ is satisfied for a.e. $t \in[0, T]$; 
2. $u(0)=u_{0}, \dot{u}(0)=v_{0}$;

3. $u \in \mathcal{C}^{1}([0, T] ; H)$ and $\ddot{u} \in L^{\infty}([0, T] ; H)$.

Proof We choose some positive integer $n$ such that $M_{1} T / n<1$ and set $h_{n}:=T / n$, $t_{i}^{n}:=i h$ for $0 \leq i \leq n$. For $0 \leq i \leq n-1$, given $u_{i}^{n}$ and $v_{i}^{n}$, we want to find $u_{i+1}^{n}, v_{i+1}^{n}$ satisfying

$$
\frac{v_{i+1}^{n}-v_{i}^{n}}{h_{n}}+f_{i}^{n} \in-N_{C\left(t_{i+1}^{n}, u_{i+1}^{n}\right)}\left(v_{i+1}^{n}\right), \quad u_{i+1}^{n}=u_{i}^{n}+h_{n} v_{i}^{n},
$$

where $f_{i}^{n} \in F\left(t_{i}^{n}, u_{i}^{n}, v_{i}^{n}\right)$. Clearly $u_{i+1}^{n}$ is defined uniquely in terms of $u_{i}^{n}$ and $v_{i}^{n}$. The first line of (9) can be rewritten as

$$
v_{i+1}^{n}-v_{i}^{n}+h_{n} f_{i}^{n} \in-N_{C\left(t_{i+1}^{n}, u_{i+1}^{n}\right)}\left(v_{i+1}^{n}\right),
$$

which is equivalent to

$$
v_{i+1}^{n}=\operatorname{proj}\left(C\left(t_{i+1}^{n}, u_{i+1}^{n}\right) ; v_{i}-h_{n} f_{i}^{n}\right) .
$$

We have the algorithm to construct the sequences $\left(u_{i}^{n}\right)_{i=0}^{n},\left(v_{i}^{n}\right)_{i=0}^{n},\left(f_{i}^{n}\right)_{i=0}^{n}$ as follows.

Algorithm

Initialization. Let $u_{0}^{n}:=u_{0}, v_{0}^{n}:=v_{0} \in C\left(0, u_{0}\right)$, choose $f_{0}^{n} \in F\left(0, u_{0}, v_{0}\right) \cap L_{F}\left(1+\left\|u_{0}\right\|+\left\|v_{0}\right\|\right) \mathbb{B}$.

Iteration. One has current points $u_{i}^{n}, v_{i}^{n}, f_{i}^{n}$. Compute $u_{i+1}^{n}:=u_{i}^{n}+h_{n} v_{i}^{n}$ and

$$
v_{i+1}^{n}:=\operatorname{proj}\left(C\left(t_{i+1}^{n}, u_{i+1}^{n}\right) ; v_{i}^{n}-h_{n} f_{i}^{n}\right) .
$$

Then, choose $f_{i+1}^{n} \in F\left(t_{i+1}^{n}, u_{i+1}^{n}, v_{i+1}^{n}\right) \cap L_{F}\left(1+\left\|u_{i+1}^{n}\right\|+\left\|v_{i+1}^{n}\right\|\right) \mathbb{B}$ and set $i:=$ $i+1$.

The algorithm is well defined thanks to $(i)$ of Assumption 1. Now we prove that the sequences $\left(u_{i}^{n}\right)_{i=0}^{n},\left(v_{i}^{n}\right)_{i=0}^{n},\left(\frac{v_{i+1}^{n}-v_{i}^{n}}{h_{n}}\right)_{i=0}^{n}$ and $\left(f_{i}^{n}\right)_{i=0}^{n}$, generated by the algorithm above, are uniformly bounded. Particularly, we show that

$$
\left\|u_{i}^{n}\right\|+\left\|v_{i}^{n}\right\| \leq M_{1}-1
$$

It is obviously true for $i=0$. Suppose that (12) holds for up to some $i \in\{0,1, \ldots, n-$ $1\}$, we will prove that (12) also holds for $i+1$. Indeed, one has $\max \left\{\left\|u_{i}^{n} \mid\right\|,\left\|u_{i+1}^{n}\right\|\right\} \leq$ $M_{1}$ and

$$
\begin{aligned}
& \left\|v_{i+1}^{n}-v_{i}^{n}+h_{n} f_{i}^{n}\right\| \leq\left\|v_{i+1}^{n}-v_{i}^{n}\right\|+h_{n}\left\|f_{i}^{n}\right\| \\
& =d\left(C\left(t_{i+1}^{n}, u_{i+1}^{n}\right) ; v_{i}^{n}\right)+h_{n} f_{i}^{n} \leq e\left(C\left(t_{i}^{n}, u_{i}^{n}\right) \cap M_{1} \mathbb{B} ; C\left(t_{i+1}^{n}, u_{i+1}^{n}\right)\right)+h_{n}\left\|f_{i}^{n}\right\| \\
& \leq \Gamma\left(t_{i}^{n}, u_{i}^{n}, t_{i+1}^{n}, u_{i+1}^{n}\right)+h_{n}\left\|f_{i}^{n}\right\| \\
& \leq h_{n} L_{C}\left(1+\left\|v_{i}\right\|\right)+h_{n}\left\|f_{i}^{n}\right\| \quad \text { (by using (i) of Assumption 1 ). }
\end{aligned}
$$


It implies that

$$
\begin{aligned}
\left\|v_{i+1}^{n}\right\| & \leq\left\|v_{i}^{n}\right\|+h_{n} L_{C}\left(1+\left\|v_{i}\right\|\right)+2 h_{n}\left\|f_{i}^{n}\right\| \\
& \leq\left\|v_{i}^{n}\right\|+h_{n} L_{C}\left(1+\left\|v_{i}\right\|\right)+2 h_{n} L_{F}\left(1+\left\|u_{i}^{n}\right\|+\left\|v_{i}^{n}\right\|\right) \\
& \leq\left\|v_{i}^{n}\right\|+h_{n}\left(L_{C}+2 L_{F}+2 L_{F}\left\|u_{i}\right\|+\left(L_{C}+2 L_{F}\right)\left\|v_{i}\right\|\right) .
\end{aligned}
$$

Consequently

$$
\begin{aligned}
& \left\|v_{i+1}^{n}\right\| \leq\left\|v_{0}\right\|+(i+1) h_{n}\left(L_{C}+2 L_{F}\right) \\
& +h_{n}\left(2 L_{F} \sum_{j=0}^{i}\left\|u_{j}\right\|+\left(L_{C}+2 L_{F}\right) \sum_{j=0}^{i}\left\|v_{j}\right\|\right) .
\end{aligned}
$$

On the other hand

$$
\left\|u_{i+1}^{n}\right\| \leq\left\|u_{i}^{n}\right\|+h_{n}\left\|v_{i}^{n}\right\| \leq \ldots \leq\left\|u_{0}\right\|+h_{n} \sum_{j=0}^{i}\left\|v_{j}\right\| .
$$

From (15) and (16), one has

$$
\left\|u_{i+1}^{n}\right\|+\left\|v_{i+1}^{n}\right\| \leq \alpha+\beta h_{n} \sum_{j=0}^{i}\left(\left\|u_{j}\right\|+\left\|v_{j}\right\|\right),
$$

where $\alpha=\left\|u_{0}\right\|+\left\|v_{0}\right\|+\left(L_{C}+2 L_{F}\right) T$ and $\beta=L_{C}+2 L_{F}+1$. Using Lemma 2.2, we obtain

$$
\left\|u_{i+1}^{n}\right\|+\left\|v_{i+1}^{n}\right\| \leq \alpha+e^{\beta i h_{n}} \leq \alpha+e^{\beta T}=M_{1}-1
$$

Consequently, by induction we have

$$
\left\|u_{i}^{n}\right\|+\left\|v_{i}^{n}\right\| \leq M_{1}-1, i=1,2, \ldots, n .
$$

On the other hand, one has

$$
\left\|f_{i}^{n}\right\| \leq L_{F}\left(1+\left\|u_{i}^{n}\right\|+\left\|v_{i}^{n}\right\|\right) \leq L_{F} M_{1}, i=1,2, \ldots, n
$$

Furthermore, from (13) one draws that

$$
\begin{aligned}
\left\|\frac{v_{i+1}^{n}-v_{i}^{n}}{h_{n}}\right\| & \leq L_{C}\left(1+\left\|v_{i}\right\|\right)+2\left\|f_{i}^{n}\right\| \leq L_{C} M_{1}+2 L_{F} M_{1} \\
& =\left(L_{C}+2 L_{F}\right) M_{1}=: M_{2}, \quad i=1,2, \ldots, n .
\end{aligned}
$$

In conclusion, the sequences $\left(u_{i}^{n}\right)_{i=0}^{n},\left(v_{i}^{n}\right)_{i=0}^{n}$ are uniformly bounded by $M_{1}$ (more precisely by $\left.M_{1}-1\right)$ and $\left(\frac{v_{i+1}^{n}-v_{i}^{n}}{h_{n}}\right)_{i=0}^{n},\left(f_{i}^{n}\right)_{i=0}^{n}$ are uniformly bounded by $M_{2}$. We 
construct the sequences of functions $\left(u_{n}(\cdot)\right)_{n},\left(v_{n}(\cdot)\right)_{n},\left(f_{n}(\cdot)\right)_{n},\left(\theta_{n}(\cdot)\right)_{n},\left(\eta_{n}(\cdot)\right)_{n}$ from $[0, T]$ to $H$ as follows: on $\left[t_{i}^{n}, t_{i+1}^{n}\right.$ [ for $0 \leq i \leq n-1$, we set

$$
u_{n}(t):=u_{i}^{n}+\frac{u_{i+1}^{n}-u_{i}^{n}}{h_{n}}\left(t-t_{i}^{n}\right), \quad v_{n}(t):=v_{i}^{n}+\frac{v_{i+1}^{n}-v_{i}^{n}}{h_{n}}\left(t-t_{i}^{n}\right),
$$

and

$$
f_{n}(t):=f_{i}^{n}, \quad \theta_{n}(t):=t_{i}^{n}, \quad \eta_{n}(t):=t_{i+1}^{n} .
$$

Then, for all $t \in] t_{i}^{n}, t_{i+1}^{n}[$

$$
\dot{u}_{n}(t)=\frac{u_{i+1}^{n}-u_{i}^{n}}{h_{n}}=v_{i}^{n} \in C\left(t_{i}^{n}, u_{i}^{n}\right), \quad \dot{v}_{n}(t)=\frac{v_{i+1}^{n}-v_{i}^{n}}{h_{n}},
$$

and

$$
\max \left\{\sup _{t \in[0, T]}\left|\theta_{n}(t)-t\right|, \sup _{t \in[0, T]}\left|\eta_{n}(t)-t\right|\right\} \leq h_{n} \rightarrow 0 \text { as } n \rightarrow+\infty
$$

The sequence $\left(v_{n}(\cdot)\right)_{n}$ is equi-Lipschitz with ratio $M_{2}$ since

$$
\left\|\dot{v}_{n}(t)\right\|=\left\|\frac{v_{i+1}^{n}-v_{i}^{n}}{h_{n}}\right\| \leq M_{2}
$$

Next we prove that the set $\Omega(t)=\left\{v_{n}(t)\right\}$ is relatively compact for all $t \in[0, T]$. Suppose to the contrary that there exists $t_{0} \in[0, T]$ such that $\Omega\left(t_{0}\right)$ is not relative compact. Then, let $3 \sigma:=\gamma\left(\Omega\left(t_{0}\right)\right)>0$. Note that $\Omega\left(t_{0}\right) \subset M_{1} \mathbb{B}$, hence $3 \sigma=$ $\gamma\left(\Omega\left(t_{0}\right)\right) \leq \gamma\left(M_{1} \mathbb{B}\right)=2 M_{1}$, which particularly implies that $\sigma \leq M_{1}$. For each $n$, we can find $i$ such that $t_{0} \in\left[t_{i}^{n}, t_{i+1}^{n}[\right.$. Then,

$$
\left\|v_{n}\left(t_{0}\right)-v_{i}^{n}\right\|=\left\|\frac{v_{i+1}^{n}-v_{i}^{n}}{h_{n}}\right\|\left\|\left(t_{0}-t_{i}^{n}\right)\right\| \leq M_{2} h_{n} .
$$

On the other hand, $v_{i}^{n} \in C\left(t_{i}^{n}, u_{i}^{n}\right) \cap M_{1} \mathbb{B} \subset C\left(t_{0}, u_{i}^{n}\right)+L_{C} h_{n} \mathbb{B} \subset C\left(t_{0}, M_{1} \mathbb{B}\right)+$ $L_{C} h_{n} \mathbb{B}$. Thus

$$
v_{n}\left(t_{0}\right) \in C\left(t_{0}, M_{1} \mathbb{B}\right)+\left(L_{C}+M_{2}\right) h_{n} \mathbb{B} .
$$

We can find $n_{0}$ large enough such that, for all $n \geq n_{0}$, we have $\left(L_{C}+M_{2}\right) h_{n}=$ $\left(L_{C}+M_{2}\right) T / n \leq \sigma$. Furthermore for all $n,\left\|v_{n}\left(t_{0}\right)\right\| \leq M_{1}$, hence

$$
\begin{aligned}
& v_{n}\left(t_{0}\right) \in\left(C\left(t_{0}, M_{1} \mathbb{B}\right) \cap\left(M_{1}+\sigma\right) \mathbb{B}\right)+\sigma \mathbb{B} \subset\left(C\left(t_{0}, M_{1} \mathbb{B}\right) \cap 2 M_{1} \mathbb{B}\right) \\
& \quad+\sigma \mathbb{B} \text { for all } n \geq n_{0} .
\end{aligned}
$$

Note that the set $C\left(t_{0}, M_{1} \mathbb{B}\right) \cap 2 M_{1} \mathbb{B}$ is relative compact (Assumption 1), hence $\gamma\left(C\left(t_{0}, M_{1} \mathbb{B}\right) \cap 2 M_{1} \mathbb{B}\right)=0$. Then, by using Lemma 2.3 , one has 


$$
\begin{aligned}
3 \sigma & =\gamma\left(\Omega\left(t_{0}\right)\right)=\gamma\left(\left\{v_{n}\left(t_{0}\right): n \geq n_{0}\right\}\right) \leq \gamma\left(\left(C\left(t_{0}, M_{1} \mathbb{B}\right) \cap 2 M_{1} \mathbb{B}\right)+\sigma \mathbb{B}\right) \\
& \leq \gamma\left(\left(C\left(t_{0}, M_{1} \mathbb{B}\right) \cap 2 M_{1} \mathbb{B}\right)\right)+\gamma(\sigma \mathbb{B})=2 \sigma,
\end{aligned}
$$

which is a contradiction. Thus the set $\Omega(t)=\left\{v_{n}(t), n \geq 1\right\}$ is relatively compact for all $t \in[0, T]$. By applying the Arzelà-Ascoli theorem (see, e.g., [10]), there exists a Lipschitz function $v(\cdot):[0, T] \rightarrow H$ with Lipschitz constant $M_{2}$ and

- $\left(v_{n}\right)$ converges strongly to $v(\cdot)$ in $\mathcal{C}([0, T] ; H)$;

- $\left(\dot{v}_{n}\right)$ converges weakly to $\dot{v}(\cdot)$ in $L^{\infty}([0, T] ; H)$.

In particular, $v(0)=v_{0}$. Let $u:[0, T] \rightarrow H, t \mapsto u(t)=u_{0}+\int_{0}^{t} v(s) d s$. Then, $u(0)=u_{0}, \dot{u}=v$ and $\ddot{u} \in L^{\infty}([0, T] ; H)$. Let us show that $u_{n}(\cdot)$ converges strongly in $\mathcal{C}([0, T] ; H)$ to $u(\cdot)$. Indeed, we have

$$
\begin{aligned}
\max _{t \in[0, T]}\left\|u_{n}(t)-u(t)\right\| & =\max _{t \in[0, T]}\left\|u_{n}(0)+\int_{0}^{t} v_{n}\left(\theta_{n}(s)\right) d s-u(0)-\int_{0}^{t} v(s) d s\right\| \\
& =\max _{t \in[0, T]}\left\|\int_{0}^{t}\left(v_{n}\left(\theta_{n}(s)\right)-v_{n}(s)+v_{n}(s)-v(s)\right) d s\right\| \\
& \leq \max _{t \in[0, T]} \int_{0}^{t}\left(M_{2}\left|\theta_{n}(s)-s\right|+\left\|v_{n}(s)-v(s)\right\| d s\right. \\
& \leq \int_{0}^{T}\left(M_{2}\left|\theta_{n}(s)-s\right|+\left\|v_{n}(s)-v(s)\right\|\right) d s \rightarrow 0,
\end{aligned}
$$

as $n \rightarrow+\infty$ since $v_{n}(\cdot)$ converges strongly to $v(\cdot)$ in $\mathcal{C}([0, T] ; H)$ and (22).

In next step, we prove that for every $t \in[0, T], \dot{u}(t) \in C(t, u(t))$. From the fact that $v_{i}^{n} \in C\left(t_{i}^{n}, u_{i}^{n}\right)$ for all $i$, we deduce for every $t \in[0, T]$ that

$$
\begin{aligned}
& v_{n}\left(\theta_{n}(t)\right) \in C\left(\theta_{n}(t), u_{n}\left(\theta_{n}(t)\right)\right) \cap M_{1} \mathbb{B} \subset C(t, u(t)) \\
& \quad+L_{C}\left\{\left|\theta_{n}(t)-t\right|+\left\|u_{n}\left(\theta_{n}(t)\right)-u(t)\right\|\right\} \mathbb{B} .
\end{aligned}
$$

It is easy to see that for every $t \in[0, T], v_{n}\left(\theta_{n}(t)\right) \rightarrow v(t)=\dot{u}(t)$ and $\left|\theta_{n}(t)-t\right|+$ $\left\|u_{n}\left(\theta_{n}(t)\right)-u(t)\right\| \rightarrow 0$ as $n \rightarrow+\infty$ because of (22) and the strongly convergence of $v_{n}(\cdot)$ to $v(t), u_{n}(\cdot)$ to $u(\cdot)$ in $\mathcal{C}([0, T] ; H)$. Since $C(t, u(t))$ is closed, we obtain that $\dot{u}(t) \in C(t, u(t))$ for every $t \in[0, T]$. It remains to prove that

$$
\ddot{u}(t) \in-N_{C(t, u(t))}(\dot{u}(t))-F(t, u(t), \dot{u}(t)) \quad \text { a.e. } t \in[0, T] .
$$

Let us define

$$
D(t):=C(t, u(t)) \cap M_{1} \mathbb{B}, \quad \forall t \in[0, T]
$$

then $D$ is nonempty, closed and convex and for all $t, s \in[0, T]$, one has

$$
d_{H}(D(t), D(s)) \leq L_{C}(|t-s|+\|u(t)-u(s)\|) \leq L_{C}\left(1+M_{1}\right)|t-s| .
$$


From (9) we have, for almost every $t \in[0, T]$, that

$$
\begin{aligned}
\dot{v}_{n}(t) & +f_{n}(t) \in-N_{C\left(\eta_{n}(t), u_{n}\left(\eta_{n}(t)\right)\right)}\left(v_{n}\left(\eta_{n}(t)\right)\right) \\
= & \left.-N_{D\left(\eta_{n}(t)\right)}\left(v_{n}\left(\eta_{n}(t)\right)\right) \text { (since }\left\|v_{n}\left(\eta_{n}(t)\right)\right\| \leq M_{1}-1\right) .
\end{aligned}
$$

Let

$$
\gamma_{n}(t):=-\left[\dot{v}_{n}(t)+f_{n}(t)\right] \text { for all } t \in[0, T] .
$$

Then, for all $c \in D\left(\eta_{n}(t)\right)$, we get

$$
\left\langle\gamma_{n}(t), c-v_{n}\left(\eta_{n}(t)\right)\right\rangle \leq 0 .
$$

Hence

$$
\sigma\left(D\left(\eta_{n}(t)\right) ; \gamma_{n}(t)\right)+\left\langle-\gamma_{n}(t), v_{n}\left(\eta_{n}(t)\right)\right\rangle \leq 0
$$

Then, integrating on $[0, T]$, one obtains

$$
\int_{0}^{T} \sigma\left(D\left(\eta_{n}(t)\right) ; \gamma_{n}(t)\right) \mathrm{d} t+\int_{0}^{T}\left\langle-\gamma_{n}(t), v_{n}\left(\eta_{n}(t)\right)\right\rangle \mathrm{d} t \leq 0 .
$$

Let us begin by estimating the second term in (29). First we prove that

$$
\int_{0}^{T}\langle\ddot{u}(t), \dot{u}(t)\rangle \mathrm{d} t=\lim _{n \rightarrow+\infty} \int_{0}^{T}\left\langle\dot{v}_{n}(t), v_{n}\left(\eta_{n}(t)\right)\right\rangle \mathrm{d} t .
$$

Indeed

$$
\begin{aligned}
\int_{0}^{T}\langle\ddot{u}(t), \dot{u}(t)\rangle \mathrm{d} t & =\frac{1}{2} \int_{0}^{T} \frac{\mathrm{d}}{\mathrm{d} t} \dot{u}^{2}(t) \mathrm{d} t=\frac{1}{2}\left(v^{2}(T)-v^{2}(0)\right) \\
& =\frac{1}{2} \lim _{n \rightarrow+\infty}\left(v_{n}^{2}(T)-v_{n}^{2}(0)\right)=\frac{1}{2} \lim _{n \rightarrow+\infty} \int_{0}^{T} \frac{\mathrm{d}}{\mathrm{d} t} v_{n}^{2}(t) \mathrm{d} t \\
& =\lim _{n \rightarrow+\infty} \int_{0}^{T}\left\langle\dot{v}_{n}(t), v_{n}(t)\right\rangle \mathrm{d} t
\end{aligned}
$$

Note that we also have

$$
\lim _{n \rightarrow+\infty} \int_{0}^{T}\left\langle\dot{v}_{n}(t), v_{n}(t)\right\rangle \mathrm{d} t=\lim _{n \rightarrow+\infty} \int_{0}^{T}\left\langle\dot{v}_{n}(t), v_{n}\left(\eta_{n}(t)\right)\right\rangle \mathrm{d} t
$$

since

$$
\int_{0}^{T}\left|\left\langle\dot{v}_{n}(t), v_{n}(t)-v_{n}\left(\eta_{n}(t)\right)\right\rangle\right| \mathrm{d} t \leq M_{2}^{2} \int_{0}^{T}\left|t-\eta_{n}(t)\right| \mathrm{d} t \rightarrow 0,
$$

as $n \rightarrow+\infty$. So we get (30). 
Since $\left\|f_{n}(t)\right\| \leq M_{2}$ for all $t \in[0, T]$, the sequence $\left(f_{n}\right)$ is bounded in $L^{\infty}([0, T] ; H)$. Therefore we can extract a subsequence, without relabeling for simplicity, converging weakly to some mapping $f(\cdot)$ in $L^{\infty}([0, T] ; H)$. On the other hand, $v_{n}\left(\eta_{n}(\cdot)\right)$ converges strongly to $\dot{u}(\cdot)$ in $L^{1}([0, T] ; H)$, so one has

$$
\left.\int_{0}^{T}\langle f(t), \dot{u}(t))\right\rangle \mathrm{d} t=\lim _{n \rightarrow+\infty} \int_{0}^{T}\left\langle f_{n}(t), v_{n}\left(\eta_{n}(t)\right)\right\rangle \mathrm{d} t .
$$

From (30) and (31), one deduces that

$$
\int_{0}^{T}\langle\dot{v}(t)+f(t), \dot{u}(t)\rangle \mathrm{d} t=\lim _{n \rightarrow+\infty} \int_{0}^{T}\left\langle-\gamma_{n}(t), v_{n}\left(\eta_{n}(t)\right)\right\rangle \mathrm{d} t .
$$

For the first term in (29), we will show that

$$
\int_{0}^{T} \sigma(D(t) ;-\dot{v}(t)-f(t)) \mathrm{d} t \leq \liminf _{n \rightarrow+\infty} \int_{0}^{T} \sigma\left(D\left(\eta_{n}(t)\right) ; \gamma_{n}(t)\right) \mathrm{d} t
$$

Let us recall that (Lemma 2.1) the convex function $x(\cdot) \mapsto \int_{0}^{T} \sigma(D(t) ; x(t)) \mathrm{d} t$ is weakly lower semicontinuous in $L^{\infty}([0, T] ; H)$ and $\gamma_{n}(t)=-\left[\dot{v}_{n}(t)+f_{n}(t)\right]$ for all $t \in[0, T]$. In addition, $\dot{v}_{n}(\cdot), f_{n}(\cdot)$ weakly converges to $\dot{v}(\cdot), f(\cdot)$ in $L^{\infty}([0, T] ; H)$, respectively. Consequently, one implies that

$$
\gamma_{n}(\cdot) \rightarrow-\dot{v}(\cdot)-f(\cdot) \text { weakly in } L^{\infty}([0, T] ; H) .
$$

Therefore

$$
\int_{0}^{T} \sigma(D(t) ;-\dot{v}(t)-f(t)) \mathrm{d} t \leq \liminf _{n \rightarrow+\infty} \int_{0}^{T} \sigma\left(D(t) ; \gamma_{n}(t)\right) \mathrm{d} t .
$$

On the other hand

$$
D(t) \subset D\left(\eta_{n}(t)\right)+L_{C}\left(1+M_{1}\right)\left|t-\eta_{n}(t)\right| \mathbb{B} \subset D\left(\eta_{n}(t)\right)+L_{C}\left(1+M_{1}\right) h_{n} \mathbb{B} .
$$

Hence

$$
\begin{aligned}
\int_{0}^{T} \sigma\left(D(t) ; \gamma_{n}(t)\right) \mathrm{d} t & \leq \int_{0}^{T} \sigma\left(D\left(\eta_{n}(t)\right) ; \gamma_{n}(t)\right) \mathrm{d} t+L_{C}\left(1+M_{1}\right) h_{n} \int_{0}^{T}\left\|\gamma_{n}(t)\right\| \mathrm{d} t \\
& \leq \int_{0}^{T} \sigma\left(D\left(\eta_{n}(t)\right) ; \gamma_{n}(t)\right) \mathrm{d} t+2 L_{C}\left(1+M_{1}\right) T M_{2} h_{n} .
\end{aligned}
$$

It leads to the following inequality

$$
\liminf _{n \rightarrow+\infty} \int_{0}^{T} \sigma\left(D(t) ; \gamma_{n}(t)\right) \mathrm{d} t \leq \liminf _{n \rightarrow+\infty} \int_{0}^{T} \sigma\left(D\left(\eta_{n}(t)\right) ; \gamma_{n}(t)\right) \mathrm{d} t
$$


From (34) and (35), we get the desired result (33). From (29), (32) and (33), we deduce that

$$
\int_{0}^{T} \sigma(D(t) ;-\dot{v}(t)-f(t)) \mathrm{d} t+\int_{0}^{T}\langle\dot{v}(t)+f(t), \dot{u}(t)\rangle \mathrm{d} t \leq 0
$$

Note that $\dot{u}(t) \in D(t)$ for every $t \in[0, T]$, we have

$$
\sigma(D(t) ;-\dot{v}(t)-f(t))+\langle\dot{v}(t)+f(t), \dot{u}(t)\rangle \geq 0 \text { a.e. } t \in[0, T] .
$$

From (36) and (37), one infers that

$$
\sigma(D(t) ;-\dot{v}(t)-f(t))+\langle\dot{v}(t)+f(t), \dot{u}(t)\rangle=0 \text { a.e. } t \in[0, T]
$$

or equivalently,

$$
\ddot{u}(t)+f(t) \in-N_{D(t)}(\dot{u}(t))=-N_{C(t, u(t))}(\dot{u}(t)) \text { a.e. } t \in[0, T] .
$$

On the other hand, one has $f_{n}(t) \in F\left(\theta_{n}(t), u_{n}\left(\theta_{n}(t)\right), v_{n}\left(\theta_{n}(t)\right)\right)$ for all $t \in[0, T]$ and $F$ is upper semicontinuous with convex and weakly compact values in $H$. Classically, we obtain that $f(t) \in F(t, u(t), \dot{u}(t))$ for almost all $t \in[0, T]$ (see, e.g., [21, Theorem V-14]). Thus

$$
\ddot{u}(t) \in-N_{C(t, u(t))}(\dot{u}(t))-F(t, u(t), \dot{u}(t)) \text { a.e. } t \in[0, T] \text {. }
$$

The result has been proved.

Remark 2 (i) It is obvious that the result is still true if we replace Assumption 1-(i) by the classical Lipschitz continuity assumption (see, e.g., [15]):

$$
\begin{gathered}
\left\|d_{H}(C(t, x) ; C(s, y))\right\| \leq L_{C}(|t-s|+\|x-y\|), \forall 0 \leq t, s \leq T \\
\text { and } x, y \in H \text { for some constant } L_{C}>0
\end{gathered}
$$

However, it is difficult for unbounded set to hold this kind of assumption, since the Hausdorff distance of two unbounded sets may equal the infinity. For example, the rotating hyperplane never satisfies (41), but satisfies Assumption 1-(i) with suitable parameters. This observation was also stated in [22], when the author studied the first-order sweeping processes with the convex moving set depending on the time. Note that in our paper, the local Lipschitz variation of the moving set is assumed in a fixed ball, while in [22], it is necessary to consider in any ball. Particularly, if $F \equiv 0$ and $0 \in C(t, x)$ for all $t \in[0, T]$ and $x \in H$, then (5) can be replaced by

$$
d_{H}\left(C(t, x) \cap M_{1} \mathbb{B} ; C(s, y) \cap M_{1} \mathbb{B}\right) \leq L_{C}(|t-s|+\|x-y\|),
$$


where $M_{1}:=\left\|u_{0}\right\|+\left\|v_{0}\right\| T$. Indeed, from (11) and $0 \in C\left(t_{i+1}, v_{i+1}^{n}\right)$ one has

$$
\left\langle v_{i}^{n}-v_{i+1}^{n}, 0-v_{i+1}^{n}\right\rangle \leq 0 \Rightarrow\left\|v_{i+1}^{n}\right\| \leq\left\|v_{i}^{n}\right\| \leq \ldots \leq\left\|v_{0}\right\| .
$$

Thus

$$
\left\|u_{i}^{n}\right\| \leq\left\|u_{i-1}^{n}\right\|+h_{n}\left\|v_{i-1}^{n}\right\| \leq \ldots \leq\left\|u_{0}\right\|+\left\|v_{0}\right\| T
$$

(ii) The compactness assumption on the moving set $C$ is also weaker than the one used in previous works [7,10,13-15] since it only requires to check the Kuratowski measure of noncompactness for a fixed ball. Furthermore, the perturbation force $F$ only needs to satisfy the weak linear growth condition.

(iii) In many applications, in practice, the set $C(\cdot)$ could be unbounded. This is the case, e.g., when $C(\cdot)$ coincides with a moving convex and closed cone. Such systems are called nonlinear complementarity systems and are of great interest in the modeling of nonregular electrical systems (see, e.g., Section 3.4 in [5]).

(iv) The compactness assumption can be replaced by the anti-monotonicity of $C$ as in [14]. Again, one does not need the boundedness and the classical Lipschitz continuity of $C$.

Theorem 3.2 Let Assumptions 1-(i), 2 hold and suppose that $-C(t, \cdot)$ is monotone for each $t \in[0, T]$. Furthermore, assume that $F$ is monotone with respect to the third variable on $\operatorname{gph}(C)$, i.e., for all $\left(t_{i}, x_{i}, y_{i}\right) \in \operatorname{gph}(C)$ and $z_{i} \in F\left(t_{i}, x_{i}, y_{i}\right)(i=1,2)$, one has

$$
\left\langle z_{1}-z_{2}, y_{1}-y_{2}\right\rangle \geq 0
$$

Then, for each initial condition, there exists a solution in the sense of Theorem 3.1.

Proof We construct the sequences $\left(u_{i}^{n}\right)_{i=0}^{n},\left(v_{i}^{n}\right)_{i=0}^{n},\left(f_{i}^{n}\right)_{i=0}^{n}$ and the sequences of functions $\left(u_{n}(\cdot)\right)_{n},\left(v_{n}(\cdot)\right)_{n},\left(f_{n}(\cdot)\right)_{n},\left(\theta_{n}(\cdot)\right)_{n},\left(\eta_{n}(\cdot)\right)_{n}$ as in Theorem 3.1. From the proof of Theorem 3.1, it is sufficient to prove the strong convergence of sequence $v_{n}(\cdot)$ in $\mathcal{C}([0, T] ; H)$. First we prove the convergence of $u_{n}(\cdot)$. For all positive integers $m \geq n$, let

$$
\varphi_{m, n}(t):=\frac{1}{2}\left\|u_{m}(t)-u_{n}(t)\right\|^{2}
$$

Then, $\varphi_{m, n}$ is differentiable almost every $t \in[0, T]$. Let $t \in[0, T]$, at which $\varphi_{m, n}$ is differentiable. Then, there exist $i, j$ such that $t \in\left[t_{i}^{m}, t_{i+1}^{m}\left[\cap\left[t_{j}^{n}, t_{j+1}^{n}[\right.\right.\right.$ and hence

$$
\frac{\mathrm{d}}{\mathrm{d} t} \varphi_{m, n}(t)=\left\langle u_{m}(t)-u_{n}(t), \dot{u}_{m}(t)-\dot{u}_{n}(t)\right\rangle=\left\langle u_{m}(t)-u_{n}(t), v_{i}^{m}-v_{j}^{n}\right\rangle .
$$

We have $v_{i}^{m} \in C\left(t_{i}^{m}, u_{i}^{m}\right) \cap M_{1} \mathbb{B} \subset C\left(t, u_{i}^{m}\right)+h_{m} L_{C} \mathbb{B}, v_{j}^{n} \in C\left(t_{j}^{n}, u_{j}^{n}\right) \subset C\left(t, u_{j}^{n}\right)+$ $h_{n} L_{C} \mathbb{B}$. From the monotonicity of $-C(t, \cdot)$ and the boundedness of $u_{i}^{m}, u_{j}^{n}$ by $M_{1}$, one has 


$$
\left\langle v_{i}^{m}-v_{j}^{n}, u_{i}^{m}-u_{j}^{n}\right\rangle \leq 2 M_{1} L_{C}\left(h_{n}+h_{m}\right) \leq 4 M_{1} L_{C} h_{n} .
$$

Hence

$$
\begin{aligned}
\frac{\mathrm{d}}{\mathrm{d} t} \varphi_{m, n}(t) & =\left\langle u_{m}(t)-u_{n}(t), v_{i}^{m}-v_{j}^{n}\right\rangle \\
& \leq\left\langle u_{m}(t)-u_{i}^{m}, v_{i}^{m}-v_{j}^{n}\right\rangle+\left\langle u_{i}^{m}-u_{j}^{n}, v_{i}^{m}-v_{j}^{n}\right\rangle+\left\langle u_{j}^{n}-u_{n}(t), v_{i}^{m}-v_{j}^{n}\right\rangle \\
& \leq 2 M_{1}^{2} h_{m}+4 M_{1} L_{C} h_{n}+2 M_{1}^{2} h_{n} \\
& \leq 4 M_{1}\left(M_{1}+L_{C}\right) h_{n},
\end{aligned}
$$

due to the $M_{1}$-Lipschitz continuity of $u_{m}(\cdot), u_{n}(\cdot)$ and the boundedness by $M_{1}$ of $u_{i}^{m}, u_{j}^{n}$. Consequently,

$$
\frac{1}{2}\left\|u_{m}(t)-u_{n}(t)\right\|^{2}=\varphi_{m, n}(t) \leq 4 M_{1} T\left(M_{1}+L_{C}\right) h_{n} \text { for all } t \in[0, T]
$$

which implies that $\left(u_{n}(\cdot)\right)_{n}$ is a Cauchy sequence in $C([0, T] ; H)$. Thus, there exists a $M_{1}$-Lipschitz function $u(\cdot)$ such that $u_{n}(\cdot)$ converges to $u(\cdot)$ uniformly and

$$
\left\|u_{n}(t)-u(t)\right\| \leq 2 \sqrt{2 M_{1} T\left(M_{1}+L_{C}\right) h_{n}} .
$$

Next we show the uniform convergence of $\left(v_{n}(\cdot)\right)_{n}$. By using (22), (43) and the Lipschitz continuity of $C, u_{n}(\cdot), v_{m}(\cdot)$, one has the following estimation

$$
\begin{aligned}
d_{C\left(\eta_{n}(t), u_{n}\left(\eta_{n}(t)\right)\right)}\left(v_{m}(t)\right) \leq & e\left(C\left(\eta_{m}(t), u_{m}\left(\eta_{m}(t)\right) \cap M_{1} \mathbb{B} ; C\left(\eta_{n}(t), u_{n}\left(\eta_{n}(t)\right)\right)\right)\right. \\
& \quad+\left\|v_{m}\left(\eta_{m}(t)\right)-v_{m}(t)\right\| \\
\leq & L_{C}\left(h_{m}+h_{n}+\left\|u_{n}\left(\eta_{n}(t)\right)-u_{m}\left(\eta_{m}(t)\right)\right\|\right)+M_{2} h_{m} \\
\leq & \left(2 L_{C}+M_{2}\right) h_{n}+L_{C}\left(\left\|u_{n}\left(\eta_{n}(t)\right)-u_{n}\left(\eta_{m}(t)\right)\right\|\right. \\
& \left.\quad+\left\|u_{n}\left(\eta_{m}(t)\right)-u_{m}\left(\eta_{m}(t)\right)\right\|\right) \\
\leq & \left(2 L_{C}+M_{2}\right) h_{n}+2 L_{C} M_{1} h_{n}+2 \sqrt{2 M_{1} T\left(M_{1}+L_{C}\right) h_{n}} .
\end{aligned}
$$

In particular, we imply that $d_{C\left(\eta_{n}(t), u_{n}\left(\eta_{n}(t)\right)\right)}\left(v_{m}(t)\right) \rightarrow 0$ as $m, n \rightarrow+\infty$. From (27) and the fact that $\left\|\dot{v}_{n}(t)+f_{n}(t)\right\| \leq 2 M_{2}$, one has

$$
\begin{aligned}
& -\dot{v}_{n}(t)-f_{n}(t) \in N_{C\left(\eta_{n}(t), u_{n}\left(\eta_{n}(t)\right)\right)}\left(v_{n}\left(\eta_{n}(t)\right)\right) \\
& =2 M_{2} \partial d_{C\left(\eta_{n}(t), u_{n}\left(\eta_{n}(t)\right)\right)}\left(v_{n}\left(\eta_{n}(t)\right)\right) .
\end{aligned}
$$

Thus

$$
\left\langle\dot{v}_{n}(t)+f_{n}(t), v_{n}\left(\eta_{n}(t)\right)-v_{m}(t)\right\rangle \leq 2 M_{2} d_{C\left(\eta_{n}(t), u_{n}\left(\eta_{n}(t)\right)\right)}\left(v_{m}(t)\right)
$$


It implies that

$$
\begin{aligned}
& \left\langle\dot{v}_{n}(t), v_{n}(t)-v_{m}(t)\right\rangle \\
\leq & \left\langle\dot{v}_{n}(t), v_{n}(t)-v_{n}\left(\eta_{n}(t)\right)\right\rangle-\left\langle f_{n}(t), v_{n}\left(\eta_{n}(t)\right)-v_{m}(t)\right\rangle \\
& +2 M_{2} d_{C\left(\eta_{n}(t), u_{n}\left(\eta_{n}(t)\right)\right)}\left(v_{m}(t)\right) \\
\leq & 2 M_{2}^{2} h_{n}-\left\langle f_{n}(t), v_{n}(t)-v_{m}(t)\right\rangle+2 M_{2} d_{C}\left(\eta_{n}(t), u_{n}\left(\eta_{n}(t)\right)\right)\left(v_{m}(t)\right) \\
= & -\left\langle f_{n}(t), v_{n}(t)-v_{m}(t)\right\rangle+\beta_{n, m}(t),
\end{aligned}
$$

where

$$
\beta_{n, m}(t):=2 M_{2}^{2} h_{n}+2 M_{2} d_{C\left(\eta_{n}(t), u_{n}\left(\eta_{n}(t)\right)\right)}\left(v_{m}(t)\right),
$$

and

$$
\left\|\beta_{n, m}\right\|_{\infty} \rightarrow 0 \text { as } m, n \rightarrow+\infty .
$$

Similarly, one has

$$
\left\langle\dot{v}_{m}(t), v_{m}(t)-v_{n}(t)\right\rangle \leq-\left\langle f_{m}(t), v_{m}(t)-v_{n}(t)\right\rangle+\beta_{m, n}(t) .
$$

As a consequence, we have, for almost every $t \in[0, T]$, that

$$
\begin{aligned}
& \left\langle\dot{v}_{m}(t)-\dot{v}_{n}(t), v_{m}(t)-v_{n}(t)\right\rangle \\
& \quad \leq-\left\langle f_{m}(t)-f_{n}(t), v_{m}(t)-v_{n}(t)\right\rangle+\beta_{m, n}(t)+\beta_{n, m}(t) \\
& \quad \leq-\left\langle f_{m}(t)-f_{n}(t), v_{m}\left(\theta_{m}(t)\right)-v_{n}\left(\theta_{n}(t)\right)\right\rangle+\alpha_{m, n}(t) \leq \alpha_{m, n}(t)
\end{aligned}
$$

where

$$
\begin{aligned}
& \alpha_{m, n}(t):=\beta_{m, n}(t)+\beta_{n, m}(t)-\left\langle f_{m}(t)-f_{n}(t), v_{m}(t)-v_{m}\left(\theta_{m}(t)\right)\right\rangle \\
& \quad-\left\langle f_{m}(t)-f_{n}(t), v_{n}\left(\theta_{n}(t)\right)-v_{n}(t)\right\rangle .
\end{aligned}
$$

The last inequality holds since

$$
\begin{aligned}
& f_{m}(t) \in F\left(\theta_{m}(t), u_{m}\left(\theta_{m}(t)\right), v_{m}\left(\theta_{m}(t)\right)\right), f_{n}(t) \in F\left(\theta_{n}(t),\right. \\
& \left.\quad u_{n}\left(\theta_{n}(t)\right), v_{n}\left(\theta_{n}(t)\right)\right),
\end{aligned}
$$

and $F$ is monotone with respect to the third variable. Note that

$$
\begin{aligned}
& \|\left\langle f_{m}(t)-f_{n}(t), v_{m}(t)-v_{m}\left(\theta_{m}(t)\right)\right\rangle+\left\langle f_{m}(t)-f_{n}(t), v_{n}\left(\theta_{n}(t)\right)\right. \\
& \left.-v_{n}(t)\right\rangle \| \leq 2 M_{2}^{2}\left(h_{m}+h_{n}\right) .
\end{aligned}
$$

Hence

$$
\left\|\alpha_{n, m}\right\|_{\infty} \rightarrow 0 \text { as } m, n \rightarrow+\infty .
$$


From (45), one has

$$
\frac{\mathrm{d}}{\mathrm{d} t}\left\|v_{m}(t)-v_{n}(t)\right\|^{2} \leq 2 \alpha_{m, n}(t) \leq 2\left\|\alpha_{n, m}\right\|_{\infty}
$$

Since $v_{m}(0)=v_{n}(0)=v_{0}$, one obtains, for all $t \in[0, T]$, that

$$
\left\|v_{m}(t)-v_{n}(t)\right\|^{2} \leq 2 T\left\|\alpha_{n, m}\right\|_{\infty} \rightarrow 0 \text { as } m, n \rightarrow+\infty
$$

It deduces that $\left(v_{n}(\cdot)\right)_{n}$ is a Cauchy sequence in $\mathcal{C}([0, T] ; H)$, which leads to the uniform convergence of $\left(v_{n}(\cdot)\right)_{n}$. Thus, the result has followed.

Theorem 3.3 (Uniqueness) Suppose that F satisfies the one-sided Lipschitz-like condition:

for all $t \in[0, T] ; x_{1}, x_{2} \in H ; y_{1} \in C\left(t, x_{1}\right), y_{2} \in C\left(t, x_{2}\right)$ and $z_{1} \in$ $F\left(t, x_{1}, y_{1}\right), z_{2} \in F\left(t, x_{2}, y_{2}\right)$, one has

$$
\left\langle z_{1}-z_{2}, y_{1}-y_{2}\right\rangle \geq-k(t)\left(\left\|x_{1}-x_{2}\right\|^{2}+\left\|y_{1}-y_{2}\right\|^{2}\right),
$$

for some function $k(\cdot) \in L^{1}([0, T] ; \mathbb{R})$. Then, for given initial condition, the sweeping process $(\mathcal{S})$ has at most a solution.

Proof Suppose that there are two solutions $u_{1}(\cdot), u_{2}(\cdot)$ of $(\mathcal{S})$ such that $u_{1}(0)=$ $u_{2}(0)=u_{0}$ and $\dot{u}_{1}(0)=\dot{u}_{2}(0)=v_{0}$. Then, there exist $f_{i}(t) \in F\left(t, u_{i}(t), \dot{u}_{i}(t)\right)$, $i=1,2$ such that

$$
\ddot{u}_{i}(t)+f_{i}(t) \in-N_{C\left(t, u_{i}(t)\right)}\left(\dot{u}_{i}(t)\right) \text { a.e. } t \in[0, T] \text {. }
$$

Using the monotonicity property of the normal cone and the condition (46), we have for almost every $t \in[0, T]$ that

$$
\left\langle\ddot{u}_{1}(t)-\ddot{u}_{2}(t), \dot{u}_{1}(t)-\dot{u}_{2}(t)\right\rangle \leq k(t)\left(\left\|u_{1}(t)-u_{2}(t)\right\|^{2}+\left\|\dot{u}_{1}(t)-\dot{u}_{2}(t)\right\|^{2}\right) .
$$

Thus

$$
\begin{aligned}
& \frac{1}{2} \frac{\mathrm{d}}{\mathrm{d} t}\left\{\left\|\dot{u}_{1}(t)-\dot{u}_{2}(t)\right\|^{2}+\left\|u_{1}(t)-u_{2}(t)\right\|^{2}\right\} \\
= & \left\langle\ddot{u}_{1}(t)-\ddot{u}_{2}(t), \dot{u}_{1}(t)-\dot{u}_{2}(t)\right\rangle+\left\langle u_{1}(t)-u_{2}(t), \dot{u}_{1}(t)-\dot{u}_{2}(t)\right\rangle \\
\leq & (k(t)+1 / 2)\left(\left\|u_{1}(t)-u_{2}(t)\right\|^{2}+\left\|\dot{u}_{1}(t)-\dot{u}_{2}(t)\right\|^{2}\right) .
\end{aligned}
$$

Then, the result follows by using Gronwall's inequality.

\section{Conclusions}

In this paper, by using tools from convex and variational analysis, the existence and uniqueness result of a class of second-order state-dependent sweeping processes in 
Hilbert space, has been studied carefully. It is remarkable that the moving set is possibly unbounded and all main assumptions (the Lipschitz continuity and the compactness of the moving set, the linear growth boundedness of the perturbation force) are weaker than the ones used in previous works, which allows more applications in practice.

Acknowledgments The authors would like to acknowledge the referees for their careful reading and insightful suggestions. The research of the second author is supported by Fondecyt Postdoc Project 3150332.

\section{References}

1. Moreau, J. J.: Rafle par un convexe variable I, Sém. Anal. Conv. Montp. Expo. 15 (1971)

2. Moreau, J. J.: Rafle par un convexe variable II, Sém. Anal. Conv. Montp. Expo. 3 (1972)

3. Moreau, J.J.: Evolution problem associated with a moving convex set in a Hilbert space. J. Differ. Equ. 26, 347-374 (1977)

4. Moreau, J.J.: Numerical aspects of the sweeping process. Comput. Methods Appl. Mech. Eng. 177, 329-349 (1999)

5. Adly, S., Haddad, T., Thibault, L.: Convex sweeping process in the framework of measure differential inclusions and evolution variational inequalities. Math. Program. Ser. B 148, 5-47 (2014)

6. Bounkhel, M., Castaing, C.: State dependent sweeping process in p-uniformly smooth and q-uniformly convex Banach spaces. Set Valued Var. Anal. 20(2), 187-201 (2012)

7. Bounkhel, M., Al-yusof, R.: First and second order convex sweeping processes in reflexive smooth Banach spaces. Set Valued Var. Anal. 18(2), 151-182 (2010)

8. Edmond, J.F., Thibault, L.: Relaxation of an optimal control problem involving a perturbed sweeping process. Math Prog. Ser. B 104, 347-373 (2005)

9. Edmond, J.F., Thibault, L.: BV solutions of nonconvex sweeping process differential inclusions with perturbation. J. Differ. Equ. 226, 135-179 (2006)

10. Kunze, M., Monteiro Marques, M.D.P.: An introduction to Moreau's sweeping process. In: Brogliato, B. (ed.) Impacts in Mechanical Systems Analysis and Modelling, pp. 1-60. Springer, Berlin (2000)

11. Castaing, C.: Quelques problèmes d'évolution du second ordre, Sém. d'Anal. Conv. Montp. 5 (1988)

12. Castaing, C., Duc Ha, T.X., Valadier, M.: Evolution equations governed by the sweeping process. Set Valued Anal. 1, 109-139 (1993)

13. Bounkhel, M.: General existence results for second order nonconvex sweeping process with unbounded perturbations. Port. Math. (N.S.) 60(3), 269-304 (2003)

14. Bounkhel, M., Azzam, D.L.: Existence results on the second-order nonconvex sweeping processes with perturbations. Set Valued Anal. 12(3), 291-318 (2004)

15. Castaing, C., Ibrahim, A.G., Yarou, M.: Some contributions to nonconvex sweeping process. J. Nonlinear Conv. Anal. 10(1), 1-20 (2009)

16. Rockafellar, R.T.: Convex Analysis. Princeton University Press, New Jersey (1970)

17. Rockafellar, R.T., Wets, R.B.: Variational Analysis. Springer, Berlin (1998)

18. Rockafellar, R.T.: Convex integral functional and duality. In: Zarantonello, E.H. (ed.) Contributions to Nonlinear Functional Analysis, pp. 215-236. Academic Press, New York (1971)

19. Deimling, K.: Multivalued Differential Equations. Walter de Gruyter, Berlin (1992)

20. Adly, S., Nacry, F., Thibault, L.: Preservation of prox-regularity of sets with application to constrained optimization. SIAM J. Optim. 26(1), 448-473 (2016)

21. Castaing, C., Valadier, M.: Convex Analysis and Measurable Multifunctions. Springer, Berlin (1977)

22. Tolstonogov, A.A.: Sweeping process with unbounded nonconvex perturbation. Nonlinear Anal. 108, 291-301 (2014) 